\title{
Comparative Study of Spiral Oblique Retinacular Ligament Reconstruction Techniques Using Either a Lateral Band or a Tendon Graft
}

\author{
Jae Yun $\mathrm{Oh}^{1}$, Jin Soo Kim¹, Dong Chul Lee ${ }^{1}$, Jae Won Yang ${ }^{1}$, Sae Hwi Ki², Byung Joon Jeon ${ }^{3}$, \\ Si Young Roh $^{1}$ \\ ${ }^{1}$ Department of Plastic and Reconstructive Surgery, Gwangmyeong Sung-Ae General Hospital, Gwangmyeong; ${ }^{2}$ Department of Plastic \\ Surgery, Inha University School of Medicine, Incheon; ${ }^{3}$ Department of Plastic Surgery, Korea University Ansan Hospital, Korea University \\ College of Medicine, Ansan, Korea
}

Background In the management of mallet deformities, oblique retinacular ligament (ORL) reconstruction provides a mechanism for automatic distal interphalangeal (DIP) joint extension upon active proximal interphalangeal joint extension. The two variants of ORL reconstruction utilize either the lateral band or a free tendon graft. This study aims to compare these two surgical techniques and to assess any differences in functional outcome. As a secondary measure, the Mitek bone anchor and pull-in suture methods are compared.

Methods A single-institutional retrospective review of ORL reconstruction was performed. The standard patient demographics, injury mechanism, type of ORL reconstruction, and pre/ postoperative degree of extension lag were collected for the 27 cases identified. The cases were divided into lateral band (group $A, n=15$ ) and free tendon graft groups (group $B, n=12$ ). Group $B$ was subdivided into the pull-in suture technique (B-I) and the Mitek bone anchor method (B-II).

Results Overall, ORL reconstructions had improved the mean DIP extension lag by $10^{\circ}(P=0.027)$. Neither the reconstructive technique choice nor bone fixation method identified any statistically meaningful difference in functional outcome $(P=0.51$ and $P=0.83$, respectively). Soft-tissue injury was associated with $30.8^{\circ}$ of improvement in the extension lag. The most common complications were tendon adhesion and rupture.

Conclusions The choice of the ORL reconstructive technique or the bone anchor method did not influence the primary functional outcome of extension lag in this study. Both lateral band and free tendon graft ORL reconstructions are valid treatment methods in the management of chronic mallet deformity.

Keywords Finger injuries / Tendons / Reconstructive surgical procedures
Correspondence: Si Young Roh Department of Plastic and Reconstructive Surgery, Gwangmyeong Sung-Ae General Hospital, 36 Digital-ro, Gwangmyeong 423-711, Korea

Tel: $+82-2-2680-7238$

Fax: +82-2-2615-7218

E-mail: psczero@gmail.com

This article was presented at the 70th Congress of the Korean Society of Plastic and Reconstructive Surgeons on November 9, 2012, in Seoul, Korea.

This study was also presented at the 30th Congress of the Korean Society for Surgery of the Hand on November 23, 2012, in Seoul, Korea.

No potential conflict of interest relevant to this article was reported.

\section{INTRODUCTION}

The lack of voluntary distal interphalangeal (DIP) extension significantly limits the overall functions of the finger with mallet deformity. The mechanical basis of this extension lag has three possible causes: 1) disruption of the terminal extensor mecha-

Copyright () 2013 The Korean Society of Plastic and Reconstructive Surgeons

This is an Open Access article distributed under the terms of the Creative Commons Attribution Non-Commercial License (http://creativecommons.org/

licenses/by-nc/3.0/) which permits unrestricted non-commercial use, distribution, and reproduction in any medium, provided the original work is properly cited.

www.e-aps.org 
nism, 2) disruption or laxity of the volar plate at the proximal interphalangeal (PIP) joint, and/or 3) contracture or spasticity of the intrinsic muscles of the hand secondary to a neuromuscular dysfunction [1].

Mallet deformity can be considered to have acute and chronic phases. In an acute setting, restoration of the extensor vector at the DIP joint is the primary concern, and this is addressed by repairing the disrupted extensor mechanism (i.e., splinting, Kwire, and tenorrhaphy). In chronic mallet deformity, however, the extension lag may result from elongated extensor elements and requires a surgical approach different from a simple repair of the DIP joint extensor mechanism.

The surgical techniques designed to address chronic mallet finger and extension lag include tendon plication, tenodermodesis, Fowler release, and oblique retinacular ligament (ORL) reconstruction, with arthrodesis as a last resort [2]. Unlike operations designed for acute mallet finger injuries, this latter group of operations has to consider the overall balance of the DIP and PIP joints. On one hand, tendon plication and tenodermodesis can improve a DIP joint extension lag but at the same time may place undue extensor stress on the PIP joint, which over time may contribute to swan-neck deformity [3]. On the other hand, Fowler release, or central slip tenotomy, allows the extensor mechanism to slide proximally around the PIP joint and does not place undue extensor stress where it is not needed [4]. Unfortunately, Fowler release is ineffective in those chronic mallet deformities with significant elongation of the extensor tendon and cannot be used in a consistent manner for all cases of chronic mallet deformity.

The concept of ORL reconstruction is a dynamic tenodesis that improves the stability of both the DIP and PIP joints by linking the volar flexor sheath to the lateral aspect of the terminal tendon, thereby providing a mechanism for automatic DIP joint extension upon active PIP extension [1]. Littler [5] first described the use of an intact lateral band for ORL reconstruction, by which the band is released proximally, rerouted to the axis of the PIP rotation on the volar side, and secured to the flexor tendon sheath. Subsequently, Thompson et al. [1] described a similar reconstruction using a free tendon of small caliber as a spiral oblique retinacular ligament (SORL). Until now, there have been no studies comparing these two ORL reconstruction techniques, and the advantage of one method over another has not been adequately explored. Additionally, the issue of whether one method of bone fixation is superior to another has not been explored in cases association with ORL repair, as Mitek bone anchors were not originally available when ORL reconstructive techniques were developed.

Hence, the purpose of this study was two-fold: 1) to statisti- cally compare the functional outcome of both reconstructive techniques, and 2) to note significant differences in the tendonto-bone fixation methods between the Mitek bone anchor and the pull-in suture technique within the free tendon graft group.

\section{METHODS}

\section{Patients}

A single-institution retrospective review was performed for all of the patients who had an extension lag/mallet deformity and underwent ORL reconstruction from August 2005 to June 2012. For each case, the usual patient demographic information (age and sex), injury mechanism, follow-up length, preoperative and postoperative extension angles, type of bone anchoring, and complications were noted.

The general indications for ORL reconstruction were 1) extension lag, 2) PIP and DIP joints with adequate passive range of motion, and 3) patient consent. Either the lateral band or free tendon graft reconstruction was chosen based on 1) an observable intact terminal tendon in the operative field, 2) a lateral band with enough strength to withstand the expected tension, and 3) the surgeon's preference.

Twenty-seven cases of mallet deformity were identified among a group of 26 patients who had undergone ORL reconstruction. Fifteen of these were reconstructed using the lateral band and were designated as group A; the remaining 12 cases of free tendon graft reconstruction were assigned to group $B$. The degree of preoperative and postoperative extension lag was measured in the neutral wrist position with active extension of the involved digit.

The collected outcomes were tabulated, and a statistical analysis was performed with SPSS ver. 19 (IBM Corp., Armonk, NY, USA). The Student's unpaired t-test was employed for continuous variables that followed a normal distribution, and a nonparametric Wilcoxon signed-rank test was used for those that followed an abnormal distribution. A P-value less than 0.05 was considered significant.

\section{Surgical techniques}

The extensor apparatus was exposed via a longitudinal incision on the dorsum of the involved finger. The intraoperative decision to use either the lateral band or a free tendon graft was made based on the factors outlined in the preceding section.

If the terminal end of the extensor tendon was available and the lateral band was intact, lateral band reconstruction was given preference (Fig. 1A). The lateral band, including a portion of the central slip of extensor digitorum, was divided up to the level of the metacarpophalangeal joint (Fig. 1B). This mobilized por- 
tion of the lateral band was then routed spirally through a volar passage that was tunneled in the fashion originally described in Thompson's publication [1]. The end of the lateral band was secured to the proximal phalangeal bone with a Mitek bone anchor (Depuy Mitek, Rayham, MA, USA) (Fig. 1C, D).

Conversely, if the terminal end of the extensor tendon was not adjacent to the lateral band or if the lateral band was damaged, the reconstruction was performed using a free tendon graft (Fig. $2 \mathrm{~A}$ ). The palmaris longus tendon is the preferable graft source, but if anatomically unavailable, plantaris or extensor digitorum longus tendons can alternately be harvested. When the tendon

\section{Fig. 1. Intraoperative photograph of lateral band technique} (group A)

(A) A midline longitudinal dorsal incision exposes the terminal extensor mechanism. (B) A healthy portion of the lateral band is mobilized for reconstruction of the oblique retinacular ligament. (C) The band is re-routed through a deep volar tunnel (not visible) and retrieved proximally on the contralateral side. (D) The lateral band is secured to the proximal phalanx (white arrow).
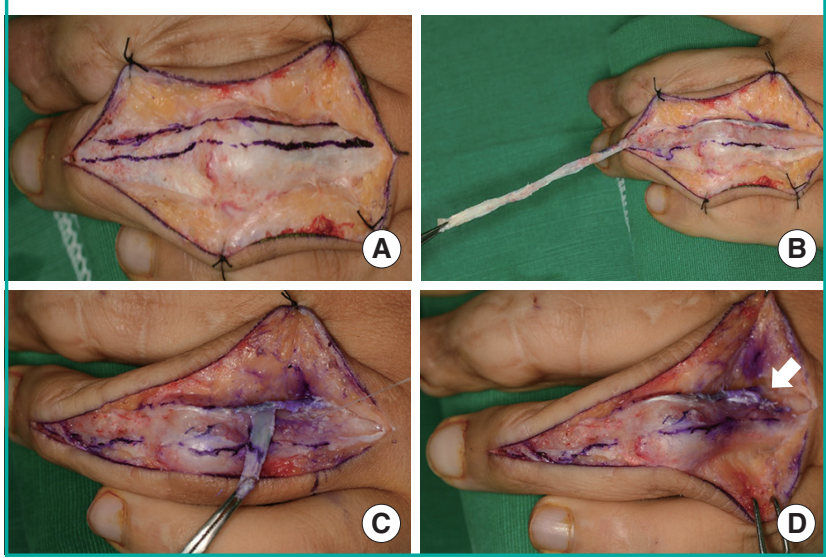

graft was ready, the distal end was fixed to the base of the distal phalanx using either a pull-in suture or a Mitek bone anchor (Fig. 2B) [6]. The proximal end of this graft was routed proximally in a spiral fashion (Fig. 2C). Prior to proximal fixation, the graft tension was adjusted to maintain a neutral extension of both the DIP and PIP joints (Fig. 2D). At this point, the proximal end of the tendon graft was secured to the aperture created in the proximal phalanx.

A conforming dressing was applied in a neutral position. Early active exercise began 3 to 7 days after the operation and continued throughout the follow-up period.

Fig. 2. Intraoperative photograph of free tendon technique (group B)

(A) The same longitudinal incision is used for free tendon ORL reconstruction. In this patient, the lateral band is attenuated and cannot be used for ORL reconstruction. (B) The distal end of a palmaris longus graft tendon is secured to the base of the distal phalanx. (C) The proximal end is routed through the volar tunnel. (D) Graft tension adjustment. The arrow points to the distal end of the graft to be secured to the proximal phalanx. ORL, oblique retinacular ligament.
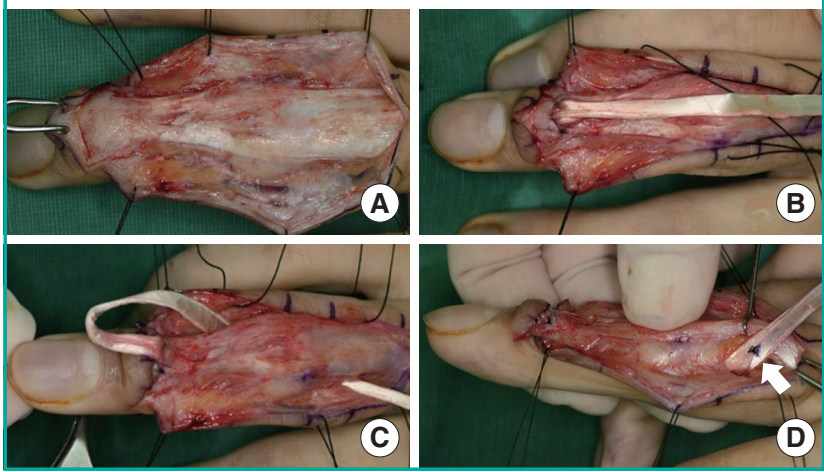

Fig. 3. Preoperative and postoperative mallet deformity

(A-C) Preoperative photograph and radiograph demonstrates DIP joint extension lag. (D-F) Postoperative views with full active DIP joint extension. White arrow points to Mitek bone anchor at the base of the distal phalanx. The arrowhead points to the site of the pull-in bone anchor. DIP, distal inter-phalangeal.
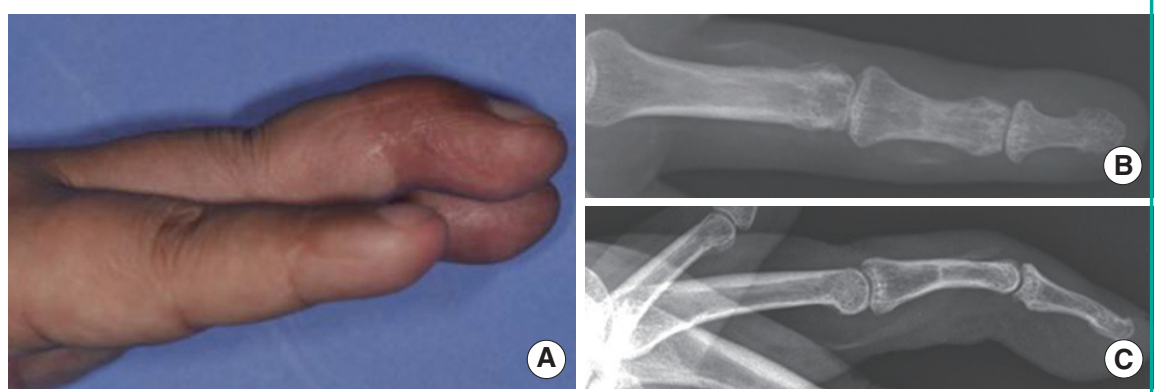

(A)
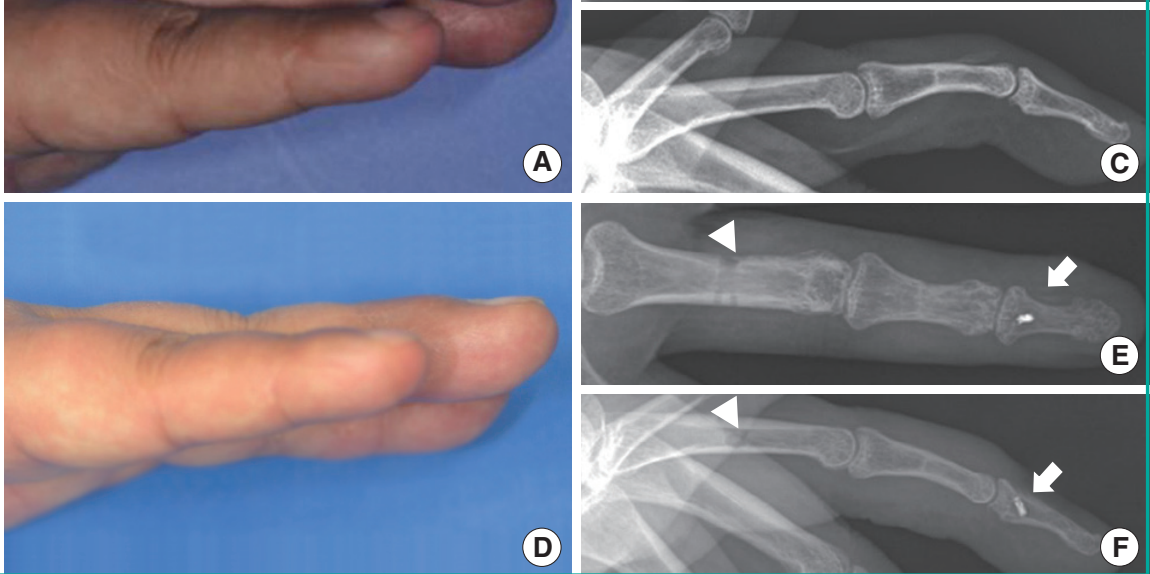


\section{RESULTS}

The mean age of the patients was 42.6 years, with a range of 21 to 70 years. As is typical for the hand injury population [7], there was a predominance of male patients ( 25 to 2 , male to female). The mean follow-up period was about 5 months. On average, the preoperative extension lag had decreased from $32.22^{\circ}$ to $22.22^{\circ}$ at follow-up visits $(\mathrm{P}=0.027)$ (Fig. 3). In group $\mathrm{A}$, the extension lag improved from $33.7^{\circ}$ to $21.3^{\circ}$, whereas in group B, it improved from $30.4^{\circ}$ to $23.3^{\circ}$. Statistically, no significant difference in the improvement of the extension lag was found between these two groups $(\mathrm{P}=0.51)$. Likewise, a statistical comparison between group B-I (pull-in suture technique) and B-II (Mitek bone anchoring) showed no significant difference $(\mathrm{P}=0.34)$ (Table 1).

Out of all 27 cases, 6 represented purely soft-tissue injuries of the extensor tendon. In this group, the preoperative extension lag was $38.3^{\circ}$, which improved to $7.5^{\circ}$ at follow-up clinic visits (Table 2).

Two types of complications observed with similar frequency in both groups were tendon adhesion and rupture. In group A, there were three cases of reoperation. Two of these cases were for tenolysis, and in the remaining case, the patient suffered from delayed tendon rupture at the site of the proximal pull-in suture. This complication was addressed by a conversion to free tendon graft reconstruction. In group B, there were two cases of reoperation. One patient underwent tenolysis, and another patient required a repeat ORL reconstruction-again, from a delayed tendon rupture at the site of the proximal pull-in suture. There were no cases of tendon rupture in which Mitek anchors had been used.

\section{DISCUSSION}

Anatomically, the ORL originates from the volar middle third of the proximal phalanx and the PIP flexor sheath, passes dorsally deep to the transverse retinacular ligament, and joins the lateral aspects of the terminal extensor tendon distally [8]. Cadaveric studies have shown that it is present in approximately half of the population [9], and the ligament has been best described as "a tiny structure, and must be looked for carefully if it is to be found at all" [10]. Functionally, this ligament appears to perform as a dynamic tenodesis, extending the DIP joint as the PIP joint is extended [1]. Although transection of this ligament alone does not cause an appreciable DIP joint extensor lag, the ORL is assumed to play a role in the coordination of the PIP and DIP joint motion and to act as a retaining ligament for the terminal tendons over the dorsum of the finger [11].

In 1967, Littler [5] described a reconstruction by which the lateral band was used to exploit the mechanism of ORL. This

\section{Table 1. Extension lag improvement by ORL reconstruction technique and by bone anchor method}

\begin{tabular}{|c|c|c|c|c|c|c|c|c|c|}
\hline \multirow[b]{2}{*}{ Group } & \multirow[b]{2}{*}{ No. of cases } & \multirow[b]{2}{*}{ Percent (\%) } & \multirow[b]{2}{*}{ Age (yr) } & \multirow[b]{2}{*}{ Sex (M:F) } & \multirow[b]{2}{*}{ Follow-up (mo) } & \multicolumn{3}{|c|}{ DIPJ extension lag $\left({ }^{\circ}\right)$} & \multirow[b]{2}{*}{ P-value } \\
\hline & & & & & & Preoperative & Postoperative & $\begin{array}{c}\text { Difference } \\
\text { (mean } \pm \text { SD) }\end{array}$ & \\
\hline A (lateral band) & 15 & 55.6 & 44.5 & $13: 2$ & 6.1 & 33.7 & 21.3 & $12.3 \pm 16.9$ & \multirow{2}{*}{$0.510(\mathrm{NS})^{\mathrm{a})}$} \\
\hline B (tendon graft) & 12 & 44.4 & 40 & $12: 0$ & 4.4 & 30.4 & 23.3 & $7.08 \pm 23.9$ & \\
\hline B-I (pull-in suture) & 7 & 25.9 & 41.9 & $7: 0$ & 5 & 34.3 & 28.6 & $5.7 \pm 23.7$ & \multirow{2}{*}{$0.827(N S)^{b)}$} \\
\hline B-II (mitek anchor) & 5 & 18.5 & 36.8 & $5: 0$ & 3.6 & 30 & 25 & $9.0 \pm 26.8$ & \\
\hline Total $(A+B)$ & 27 & 100 & 42.6 & $25: 2$ & 5.33 & $32.2 \pm 14.9$ & $22.2 \pm 18.4$ & 10 & $0.015^{c)}$ \\
\hline
\end{tabular}

Table 2. Extension lag improvement among soft tissue injury patients

\begin{tabular}{|c|c|c|c|c|c|c|c|}
\hline \multirow{2}{*}{ Case } & \multirow{2}{*}{ Sex } & \multirow{2}{*}{ Age (yr) } & \multirow{2}{*}{ Finger } & \multirow{2}{*}{ Duration of deformity (mo) } & \multicolumn{3}{|c|}{ DIPJ extension lag $\left({ }^{\circ}\right)$} \\
\hline & & & & & Preoperative & Postoperative & Difference \\
\hline 1 & M & 50 & Ring & 40 & 55 & 25 & 30 \\
\hline 2 & M & 30 & Long & 7 & 30 & 10 & 20 \\
\hline 3 & M & 29 & Long & 6 & 40 & 0 & 40 \\
\hline 4 & M & 60 & Index & 8 & 45 & 10 & 35 \\
\hline \multirow[t]{2}{*}{$5^{\text {a) }}$} & M & 32 & Long & 14 & 15 & 0 & 15 \\
\hline & - & - & Index & 20 & 45 & 0 & 45 \\
\hline Total & - & 40.2 & - & 15.8 & 38.3 & 7.5 & 30.83 \\
\hline
\end{tabular}


procedure was able to restore the balance of extensor forces across both interphalangeal joints but required an intact, healthy terminal tendon at the extensor insertion site. Thus, its application was limited to those mallet deformities where the lateral band was found to be robust at the time of intraoperative inspection.

This deficiency in lateral band ORL reconstruction was addressed in a subsequent 1978 report by Thompson et al. [1], in which a free tendon graft was used to replace the lateral band. The use of a free tendon graft had several advantages over Littler's initial procedure. First, the operation was possible even if the terminal extensor tendon was ruptured. Second, the terminal extension could be restored without altering the extensor mechanism. Third, the extensor tension could be adjusted by a wider margin because of the additional length afforded by the graft tendon. The obvious disadvantage of this procedure was the requirement for a free tendon graft, along with the usual comorbidities associated with such an operation.

In this study on chronic mallet deformity, the goal was to assess whether the use of a lateral band or free tendon graft would have any influence on the outcome. Overall, the results support the use of both forms of the reconstruction technique. On average, the mean extension lag improved by 12.4 degrees in the lateral band group (group A) and by 7.1 degrees in the free tendon graft group (group B). However, the difference between these two groups was not significant (Table 1). This outcome is not surprising when considering that these procedures were designed upon the same biomechanical basis.

In the subgroup analysis, there were no significant differences between the Mitek bone anchor group (B-I) and the pull-in suture group (B-II) when comparing the extension lag improvement $\left(5.7^{\circ}\right.$ vs. $\left.9.0^{\circ}, \mathrm{P}=0.83\right)$. However, the method of tendon fixation cannot be trivialized in the discussion of ORL reconstruction for the following reason: the fixation method dictates the duration of immobilization and the timing of physical therapy.

The overall improvement of approximately $10^{\circ}$ can be challenged in the interest of the operation. This compares to a 1987 publication by Kleinman and Petersen [12] in which 12 ORL reconstructions were evaluated and shown to improve DIP extension to normal angles $\left(0^{\circ}\right)$ in nine cases. The difference arises from the prevalence of injury mechanisms. Whereas soft-tissue injuries were predominant ( 9 out of 12) in that study, our series represented bony injuries in 19 cases out of 27 . The remaining 6 cases were accompanied by soft-tissue injuries only, and a review of these cases revealed that ORL reconstruction was able to improve extension lag by approximately $31^{\circ}$ on average. If the prevention of swan-neck deformity were not taken into consideration, a relative contraindication could be made for the presence of bony injuries because ORL reconstruction is associated with minimal improvement in extension lag in the context of accompanying fractures.

In Littler's initial publication, the freed portion of the lateral band was fixed to the fibrous tendon sheath, and in the subsequent publication, pull-out tenodesis was described in both the proximal and distal phalanxes, using either hemoclips or buttons. Active motion exercises were commenced at 3 weeks in both of these studies $[1,3]$. In a 1984 publication on ORL reconstruction, Kleinman and Petersen [12] reported the fixation of the distal end by a pull-out button anchor and the proximal end to the fibrous rim of fibroosseous canal, with K-wire fixation of the DIP joint. Again, the rehabilitation was delayed until three weeks after the operation. At our institution, the tendon ends were directly fixed to the bone in either ORL reconstructive technique. Because of this, prolonged immobilization was unnecessary, and active motion exercise could be commenced early, within 3 to 7 days after the operation.

The two cases of tendon ruptures occurred when the pullin suture method was used to anchor the proximal end of the tendon. This compares to zero incidence of tendon rupture with Mitek bone anchors. Though this study was not designed to statistically discriminate between the complication rates of these two fixation methods, the fact that tendon rupture was associated only with the pull-in suture was a clinically significant finding.

In conclusion, this study supports continued use of ORL reconstruction with certain caveats. The two ORL reconstructive techniques did not show statistically significant differences in terms of functional outcome of DIP extension, though softtissue injuries were associated with greater improvement. In the subgroup analysis, the method of bone anchoring did not reveal significant differences in the primary outcome of extension lag, but the complication of tendon rupture with the pull-in suture does warrant further inquiry into the method of tendon fixation in ORL reconstruction.

\section{REFERENCES}

1. Thompson JS, Littler JW, Upton J. The spiral oblique retinacular ligament (SORL). J Hand Surg Am 1978;3:482-7.

2. Makhlouf VM, Deek NA. Surgical treatment of chronic mallet finger. Ann Plast Surg 2011;66:670-2.

3. Ay S, Akinci M, Ercetin O. The Brooks and Graner procedure for treatment of chronic tendinous mallet finger deformity. Tech Hand Up Extrem Surg 2004;8:21-4.

4. Chao JD, Sarwahi V, Da Silva YS, et al. Central slip tenoto- 
my for the treatment of chronic mallet finger: an anatomic study.J Hand Surg Am 2004;29:216-9.

5. Littler JW. The finger extensor mechanism. Surg Clin North Am 1967;47:415-32.

6. Kim JW, Chung SM. Pull-in suture technique for the disinsertion of the phalangeal tendon distal insertion. J Korean Soc Plast Reconstr Surg 2008;35:723-8.

7. Chung KC, Spilson SV. The frequency and epidemiology of hand and forearm fractures in the United States. J Hand Surg Am 2001;26:908-15.

8. Wehbe MA. Anatomy of the extensor mechanism of the hand and wrist. Hand Clin 1995;11:361-6.
9. Shrewsbury MM, Johnson RK. A systematic study of the oblique retinacular ligament of the human finger: its structure and function.J Hand Surg Am 1977;2:194-9.

10. Harris C Jr, Rutledge GL Jr. The functional anatomy of the extensor mechanism of the finger. J Bone Joint Surg Am 1972;54:713-26.

11. Schweitzer TP, Rayan GM. The terminal tendon of the digital extensor mechanism: Part II, kinematic study. J Hand Surg Am 2004;29:903-8.

12. Kleinman WB, Petersen DP. Oblique retinacular ligament reconstruction for chronic mallet finger deformity. J Hand Surg Am 1984;9:399-404. 